liver disease were excluded (90 LTs). Our database showed 232 primary LTs between 2000 and 2020 with the following distribution :195 elective LTs for ESCLD and 37 superurgent LTs for ALF. Recipients' age and weight were significantly higher in ALF group. Most common indication for LT in ESCLD group was biliary atresia while seronegative

\begin{tabular}{|c|c|c|c|}
\hline Parameter & $\begin{array}{l}\text { ALF group } \\
(n=37)\end{array}$ & $\begin{array}{l}\text { ESCLD group } \\
(n=195)\end{array}$ & $\mathbf{P}$ \\
\hline \multicolumn{4}{|l|}{ Recipient gender: } \\
\hline - Male & $16(43.2 \%)$ & $102(52.3 \%)$ & 0.312 \\
\hline - Female & $21(56.8 \%)$ & $93(47.7 \%)$ & \\
\hline \multicolumn{4}{|l|}{ Recipient age at transplant (years): } \\
\hline Median (Min. - Max.) & $8.8(0.1-16.7)$ & $2.5(0.3-17.2)$ & 0.031 \\
\hline \multicolumn{4}{|l|}{ Recipient Weight (kg) } \\
\hline Median (Min. - Max.) & $25.5(2.7-66.5)$ & $12.5(4.7-89)$ & 0.011 \\
\hline \multicolumn{4}{|l|}{ Waiting time(days) } \\
\hline Median (Min. - Max.) & $3(1-41)$ & $60.5(1-560)$ & $<0.001$ \\
\hline \multicolumn{4}{|l|}{ Graft type } \\
\hline - Graft variant & $23(62.2 \%)$ & $155(79.4 \%)$ & 0.016 \\
\hline - Whole graft & $14(37.8 \%)$ & $38(19.4 \%)$ & \\
\hline - Missing & 0 & $2(1.2 \%)$ & \\
\hline \multicolumn{4}{|l|}{ Donor gender: } \\
\hline - Male & $45.7 \%$ & $46.9 \%$ & 0.902 \\
\hline - Female & $54.3 \%$ & $53.1 \%$ & \\
\hline \multicolumn{4}{|l|}{ Donor weight $(\mathrm{kg})$} \\
\hline Median(min-max) & $67(8-90)$ & $68(10-98)$ & 0.912 \\
\hline \multicolumn{4}{|l|}{ Donor age (years) } \\
\hline Median (Min. - Max.) & $39(0.9-65)$ & $29.5(1-66)$ & 0.039 \\
\hline \multicolumn{4}{|l|}{ Vascular complications } \\
\hline \multicolumn{3}{|l|}{ - Massive retroperitoneal hematoma } & $\mathrm{FEp}=1.000$ \\
\hline from femoral vein bypass cannula. & $0(0 \%)$ & $1(0.5 \%)$ & $\mathrm{FEp}=1.000$ \\
\hline - HAS & $2(5.4 \%)$ & $13(6.7 \%)$ & $\mathrm{FEp}=1.000$ \\
\hline - PVS & $0(0 \%)$ & $14(7.2 \%)$ & FEp $=0.134$ \\
\hline$\bullet$ HAT & $1(2.7 \%)$ & $10(5.1 \%)$ & $\mathrm{FEp}=1.000$ \\
\hline$\bullet$ PVT & $1(2.7 \%)$ & $7(3.6 \%)$ & FEp $=1.000$ \\
\hline - HVS & $1(2.7 \%)$ & $0(0 \%)$ & FEp $=0.159$ \\
\hline \multicolumn{4}{|l|}{ Biliary complications } \\
\hline - CHD sludge & $0(0 \%)$ & $2(1 \%)$ & FEp $=1.000$ \\
\hline - Biliary stricture & $3(8.1 \%)$ & $21(10.8 \%)$ & FEp $=0.775$ \\
\hline - Bile leak & $0(0 \%)$ & $23(11.8 \%)$ & $\mathrm{FEp}=0.031$ \\
\hline \multicolumn{4}{|l|}{ Rejection: } \\
\hline & $17(45.9 \%)$ & $62(31.8 \%)$ & 0.096 \\
\hline Cause of graft loss & $(n=5)$ & $(n=27)$ & \\
\hline • HAT & $0(0 \%)$ & $9(33.3 \%)$ & FEp $=0.288$ \\
\hline - PNF & $2(40 \%)$ & $5(18.5 \%)$ & FEp $=0.296$ \\
\hline - Chronic rejection & $3(60 \%)$ & $6(22.2 \%)$ & FE $p=0.121$ \\
\hline - Biliary tract complications & $0(0 \%)$ & $7(25.9 \%)$ & FEp $=0.560$ \\
\hline Cause of death & $(n=9)$ & $(n=17)$ & \\
\hline Unknown & $0(0 \%)$ & $5(29.4 \%)$ & FE $p=0.129$ \\
\hline Cardiopulmonary & $3(33.3 \%)$ & $1(5.9 \%)$ & FEp $=0.104$ \\
\hline Cerebral oedema & $1(11.1 \%)$ & $0(0 \%)$ & FEp $=0.346$ \\
\hline Fungal infection & $1(11.1 \%)$ & $0(0 \%)$ & FEp $=0.346$ \\
\hline Gastro-intestinal & $0(0 \%)$ & $1(5.9 \%)$ & FE $p=1.000$ \\
\hline Intra-cranial hemorrhage & $1(11.1 \%)$ & $0(0 \%)$ & FEp $=0.346$ \\
\hline Liver Failure & $1(11.1 \%)$ & $4(23.5 \%)$ & $\mathrm{FEp}=0.628$ \\
\hline Recurrence of disease & $0(0 \%)$ & $1(5.9 \%)$ & $\mathrm{FEp}=1.000$ \\
\hline Sepsis & $2(22.2 \%)$ & $5(29.4 \%)$ & $\mathrm{FEp}=1.000$ \\
\hline
\end{tabular}

hepatitis was the most common indication in ALF group. Time on transplant waiting list was significantly shorter for ALF group. Regarding pre-transplant location, home location was higher in ESCLD group while hospital and PICU location were significantly higher in ALF group. In terms of the source of the graft, living donors were significantly higher in the ESCLD group (34 donors) than ALF group where no living donors where used. There was no statistically significant difference between both groups in terms of rejection and vascular complications while biliary complications showed significantly higher bile leak rates in the ESCLD group. Post-transplant survival was significantly higher in the ESCLD group as 1-,5- and 10 years survival rates for ESCLD group were 97.9\%,93.9\%,89.4\%,85.0\% respectively while survival rates in ALF recipients during the same period were fixed at $78.3 \%(\mathrm{P}=0.007)$. Graft survival was longer in the ESCLD group but the difference was not statistically significant.

Summary We studied 195 elective LTs for patients with ESCLD and 37 super-urgent LTs for ALF patients. Recipients in ALF group were significantly older and heavier. Vascular complications and rejection rates did not show significant difference between two groups while bile leak was significantly higher in the ESCLD group. Patient survival was significantly higher in ESCLD group while graft survival did not show significant difference between two groups.

Conclusion Post LT survival in ALF patients is significantly inferior to ESCLD patients.

\section{P37 PARENTERAL INTAKE OF VITAMINS AND TRACE ELEMENTS IN CHILDREN ON HOME PARENTERAL NUTRITION: CAN WE MEET ESPGHAN RECOMMENDATIONS USING FIXED-DOSE MULTIVITAMIN/TRACE ELEMENT PRODUCTS? - EXPERIENCES FROM A REGIONAL PAEDIATRIC INTESTINAL FAILURE SERVICE}

Claire Leivers, Kai Hensel, Camilla Salvestrini, Emma Williams. Cambridge University Hospitals NHS Trust

\subsection{6/flgastro-2021-bspghan.46}

Background ESPGHAN guidelines stipulate the recommended quantities of parenteral vitamins and trace elements to be used in children on home parenteral nutrition (HPN). The use of standard fixed-dose multivitamin/multitrace products makes tailoring the individual intake of these nutrients challenging in daily clinical practice. Importantly, the dosage of these products received is based on an individual's body weight and is highly influenced by other factors, such as number of HPN bags infused per week and HPN composition, and their intake not routinely assessed.

Objective The aim of this study was to assess parenteral vitamin and trace element receipt in children receiving HPN, in light of the recently updated 2018 ESPGHAN recommendations.

Methods The average daily parenteral intake of vitamins and trace elements of the paediatric HPN cohort at Cambridge University Hospitals (CUH) was assessed and expressed as a percentage of the current ESPGHAN recommendations. This was adjusted for, where HPN was not meeting the child's full parenteral energy requirements. Where ESPGHAN stated 
maximum quantities, the percentage of this quantity was calculated.

Results A total of 12 patients aged between one and 14 years were included. All children met adjusted parenteral recommendations for water soluble vitamins except for vitamin C, where intake was suboptimal in one case. In all patients, vitamin A recommendations were exceeded, whereas vitamins D and $\mathrm{K}$ were suboptimal. Recommendations for zinc were clearly surpassed in all children, but this was below the maximum quantity advised. Iodine was adequately supplied in $50 \%$, copper in $67 \%$ and selenium in $75 \%$ of patients. No patient exceeded the maximum recommended intake of vitamin $\mathrm{E}$ and chromium. The maximum dose of manganese was exceeded in one patient.

Conclusion Licensed parenteral multivitamin/multitrace products in the UK provide fixed combinations of multiple micronutrients and are dosed based on weight, although recommended intakes are mostly expressed as fixed daily quantities. Consequently, meeting the ESPHAN recommendations with these products is challenging and monitoring of serum concentrations of these nutrients is essential in this vulnerable patient population.

\section{P38 PEDIATRIC INFLAMMATORY BOWEL DISEASE AND HIDRADENITIS SUPPURATIVE: A CHALLENGING ASSOCIATION?}

Mark Mahon, Daniela Levanon. Jacobi Medical Center/Albert Einstein

10.1136/flgastro-2021-bspghan.47

Introduction Within Inflammatory Bowel Disease (IBD), perineal lesions are a common extra-intestinal manifestation, yet may mask other entities. Including several etiologies across a number of subspecialties, most of which are better appreciated in adulthood. This report focuses on an unusual dermatological association with IBD, presenting at an atypical time in the disease course.

Case Report 11-year-old obese Hispanic female presented with the chief complaint of epistaxis and was noted to have painful lower extremity nodules consistent with erythema nodosum and gluteal cleft lesions. Review of systems revealed fatigue, anorexia and diarrhea for two months prior accompanied by a $6.8 \mathrm{~kg}$ weight loss over that period. On admission, she was febrile $\left(38.3^{\circ} \mathrm{C}\right)$, tachycardic and hypotensive with baseline laboratory values notable for leukocytosis (13.1/ $\mathrm{nL}$ ), hypochromic microcytic anemia (hemoglobin $10.3 \mathrm{~g} / \mathrm{dL}$ and mean corpuscular volume $70.3 \mathrm{fL}$ ), thrombocytopenia $(55 / \mathrm{nL})$ and evidence of systemic inflammation with elevated CRP/ESR.

Stool studies were significant for fecal leukocytes and a CT revealed mural thickening with fat stranding; segmental colitis was confirmed on colonoscopy. At that time, a punch biopsy of the gluteal lesion revealed granulomatous dermatitis, presumed to be cutaneous Crohn's Disease (CD). Treatment was initiated with Metronidazole and Methylprednisolone. Soon after the clinical course became complicated by the development of a rectovaginal fistula. Induction and maintenance treatment were achieved with Infliximab and the patient was discharged with the diagnosis of CD with perineal involvement.

Multidisciplinary team monitoring over the following three years ensued before the patient reported progression of intertriginous lesions, this time to the axillae and infra-mammary areas. Punch biopsy at the new lesion established the diagnosis of Hidradenitis Suppurativa (HS), with a pathological confirmation.

Discussion HS is a chronic inflammatory dermatological disease of the apocrine glands, characterized by recurrent and painful, deep-seated nodules, abscesses, sinus tracts and/or fistulas. It affects inverse areas of the skin following the distribution of apocrine glands. Prevalence is higher post-puberty, with smoking and obesity acting as risk factors. The association with IBD, particularly $\mathrm{CD}$ is stronger in the severe phenotype and in pancolitis. The formal diagnosis is made on average one decade after the onset of IBD. Up to $25 \%$ of IBD patients experience extra-intestinal manifestations, perineal pathology accounts for $50 \%$ of the cases. Yet, in the absence of extra-perineal intertriginous involvement, the possibility of HS may be less recognizable. Improved awareness to this association among Paediatricians and Paediatric Gastroenterologists is important as co-pathology may require treatment escalation to immunosuppressive agents or alterations to monoclonal antibody regimen. More intensive treatment is often required as disease remission is harder to achieve for HS than CD.

Conclusion HS, when associated with CD, typically occurs one decade after the initial IBD diagnosis. This case presents a much shorter interval between such diagnoses, and potentially a dual presentation. This has not been appreciated in the literature to date and possibly suggests rare but earlier association when present. Analysis of a larger pediatric IBD cohort with HS will help clarify the pattern of association.

\section{P39 PHENOTYPE FLIP - RISK OF DEVELOPING CROHN'S DISEASE FOLLOWING RESTORATIVE PROCTO- COLECTOMY FOR ULCERATIVE COLITIS}

Alison Campbell, Bruce Jaffray. Great North Children's Hospital, Newcastle

\subsection{6/flgastro-2021-bspghan.48}

Introduction $30 \%$ of Ulcerative Colitis (UC) patients require surgery. Restorative procto-colectomy (RPC) with ileal pouch-anal anastomosis (IPAA) is the preferred procedure. Published experience suggests up to $25 \%$ of patients subsequently manifest Crohn's Disease (CD), and 50\% of these require pouch excision. Despite long-term follow up, we had not previously identified Crohn's conversion in our UC pouch patients.

Aim We analysed our UC cohort, for cases where the pouch was either excised or de-functioned. The aim of this study was to quantify the incidence of $\mathrm{CD}$ in this group.

Methods All children undergoing pouch surgery have had data regarding their surgery, pre-op management and disease status recorded contemporaneously. This database was interrogated and further results from the histology database retrieved. In addition, pre-pouch surgery work up, specifically diagnosis and number of pre-op colonoscopies, was evaluated.

Results From 1999 to 2020, 84 children (mean age 13.5 yrs) have undergone surgery for UC with the intention of performing RPC and IPAA. 3 were unable to be anastomosed at initial surgery; 1 subsequently underwent successful IPAA, 2 have end ileostomies. 